\title{
Gait pattern and cognitive performance during treadmill walking in parkinson disease
}

\author{
Helena Fernández-Lago, Olalla Bello, Virginia López-Alonso, José Andrés Sánchez, \\ Luis Morenilla, Miguel Ángel Fernández-del-Olmo
}

\begin{abstract}
Objective: The aim of this study was to explore whether attentional demands are involved in gait improvements in Parkinson disease (PD) patients when they walk on a treadmill.

Design: Nineteen individuals with idiopathic PD and 19 age-matched healthy controls participated in this study. Participants walked on a treadmill and on overground under single task (walk only) and dual task (walk performing a simultaneous cognitive task) conditions. The dual-task paradigm was used to reveal the attention allocation behavior. Gait pattern and cognitive performance was measured.

Results: The PD group showed reduced gait variability when walking on a treadmill in comparison with overground. However, this reduction did not deteriorate during the dual task. Moreover, there were no differences in the cognitive performance between treadmill and overground walking.

Conclusions: This study does not support the proposition attentional resource allocation as a possible mechanism for the treadmill-associated gait improvements observed in PD.
\end{abstract}

Key Words

Dual task; Gait; Parkinson disease; Treadmill

Gait disorders are among the most significant impairments in Parkinson disease (PD) that severely affect the individual's quality of life. ${ }^{1}$ PD gait is characterized by a reduced gait speed, increased strideto-stride variability, and a specific difficulty to regulate stride length. ${ }^{1}$ In addition, people with PD suffer a deficit in executive functions and attention abilities (see Dirbenger and Jahanshahi ${ }^{2}$ for an extensive review of executive deficits in PD and Yogev-Seligmann et al. ${ }^{3}$ for a review of the role of executive function and attention in gait). These cognitive dysfunctions could explain the exacerbated gait impairments in PD subjects (ie, reduced walking speed and stride length, and increased stride-to-stride variability) when they perform a concurrent task. ${ }^{4-6}$ Previous studies have examined the relationship between cognition and gait performance in people with PD using a secondary task during walking (dualtask paradigm). ${ }^{4,-10}$ Dual tasking (DT) is a component of executive function, which refers to the ability to allocate attention to tasks that are performed at the same time. ${ }^{11}$ Although several theories have been proposed to explain DT interference, all of them agree that when two tasks are performed concurrently, performance decrements are observed in one or both tasks. ${ }^{10,11}$ For instance, and according to the capacity sharing model, ${ }^{5,12}$ when two attentionally demanding tasks are performed simultaneously, competition of attentional resources results in the deterioration of performance in one or both of the tasks. Therefore, it is important to explore the role of attention associated with therapeutic strategies to improve the gait in subjects with PD.

In the last decade, several studies have shown the therapeutic use of treadmill training for gait rehabilitation in PD. ${ }^{13}$ Several weeks of treadmill training lead to an improvements in several gait parameters such as enlarged stride length, increased speed, decreased double support, reduced swing time variability, and reduced stride length variability between others. ${ }^{12,14-16}$ Notably, subjects with PD increased their stride length after 5 weeks of treadmill training but not when the training consisted of walking overground. ${ }^{14} \mathrm{~A}$ recent study showed improvements in walking speed and walking endurance even 6 months after the treadmill training. ${ }^{15}$ In addition to these long-term effects of treadmill training, immediate improvements on overground gait, such as faster speed and longer step length, have been reported after a single treadmill session in PD. ${ }^{17}$ 
The therapeutic effect of treadmill could be explained as a specific gait modulation in PD subjects when walking over this device. PD subjects walk on a treadmill with lower stride-to-stride variability ${ }^{18}$ and higher length step in comparison with walking overground. ${ }^{17}$ However, the mechanisms underlying these positive treadmill effects in PD remain unknown even though several theories have been postulated. ${ }^{19,20}$ One of these theories refers to attentional resources. Although regulation of gait pattern is an automatic process that does not require attention in healthy adults, ${ }^{4}$ people with PD are able to improve their gait when they direct their attention to their walking movement, ${ }^{21,22}$ suggesting an impairment in the automatic control of the gait. Thus, it is plausible that the stable environment and absence of distracters associated with walking over the treadmill allow PD patients to allocate attention to their gait in comparison with an overground walking condition. However, to the best of our knowledge, there are no studies that have explored the role of attention during treadmill walking in subjects with PD.

Therefore, the main goal of this study was to investigate whether attention could be an underlying mechanism for the gait improvements observed during treadmill walking in PD. To this end, and using a dual-task paradigm, the present work compared the gait pattern in PD and controls when walking over the treadmill and when walking overground during the performance of a concurrent cognitive task. The proposed hypothesis was that if the gait improvements observed while walking over a treadmill depend on attentional resources, performance of a cognitive task will lead to impairment in one or both of the tasks.

\section{Methods}

Subjects

Nineteen individuals diagnosed with idiopathic PD by a neurologist, according to the United Kingdom Bank Criteria $^{23}$ (11 males and 8 females, mean age $\left.=59.79 \pm 12.63\right)$, and 19 age-matched healthy controls ( 11 males and 8 females, mean age $=59.53 \pm 12.1)$ were recruited for the study from a local community. Inclusion criteria for participants with PD was diagnosis of idiopathic PD, the ability to walk for 10 minutes without stopping or walking assistance, absence of neurologic disorders other than PD, and absence of orthopedic, cardiovascular, or visual disturbances that could affect gait. Healthy controls were included if they did not have a history of neurological pathology or other disease that could affect the ability to walk. All participants did not use a treadmill for at least 12 months before the experiment. No participant showed dementia as assessed by a mini-mental state examination (MMSE). The level of severity of motor signs associated with PD was measured in ON state $(45 \mathrm{~min}-1.5 \mathrm{~h}$ after medication intake) using the Unified Parkinson's Disease Rating ScalePart-III ${ }^{24}$ (UPDRS-III) and Hoehn and Yahr scale ${ }^{25}$ (H\&Y). A neurologist confirmed the ON state. All participants gave their written informed consent according to the declaration of Helsinki (1964) before entering the study. The experimental procedures were approved by the local ethics committee.

\section{Apparatus}

A treadmill with handrails (SportsArt 6300; Sports Arts Fitness) was used. Gait performance overground and on the treadmill was recorded using an optical detection system (OptoGait; Microgait, USA). This optical and modular system included transmitting and receiving bars of infrared LEDs. The apparatus detected the interruptions of the communication between the bars during walking. Wireless headphones (Philips Hi.Fi, SHD8600UG) were used for the cognitive task.

\section{Testing Procedure}

All participants were tested in four conditions: overground single task (ST: walk only), overground dual task (DT: walk and perform a cognitive task), treadmill ST, and treadmill DT. Single and dual task conditions were arranged in a random order. However, overground and treadmill conditions were not counterbalanced because it has been reported that treadmill walking has significant and long-lasting effects on the speed and frequency of consequent overground walking. ${ }^{17}$ The experimental sessions were carried out while patients were ON medication, confirmed by a neurologist evaluating the motor items of the UPRDS scale (Fig. 1). 


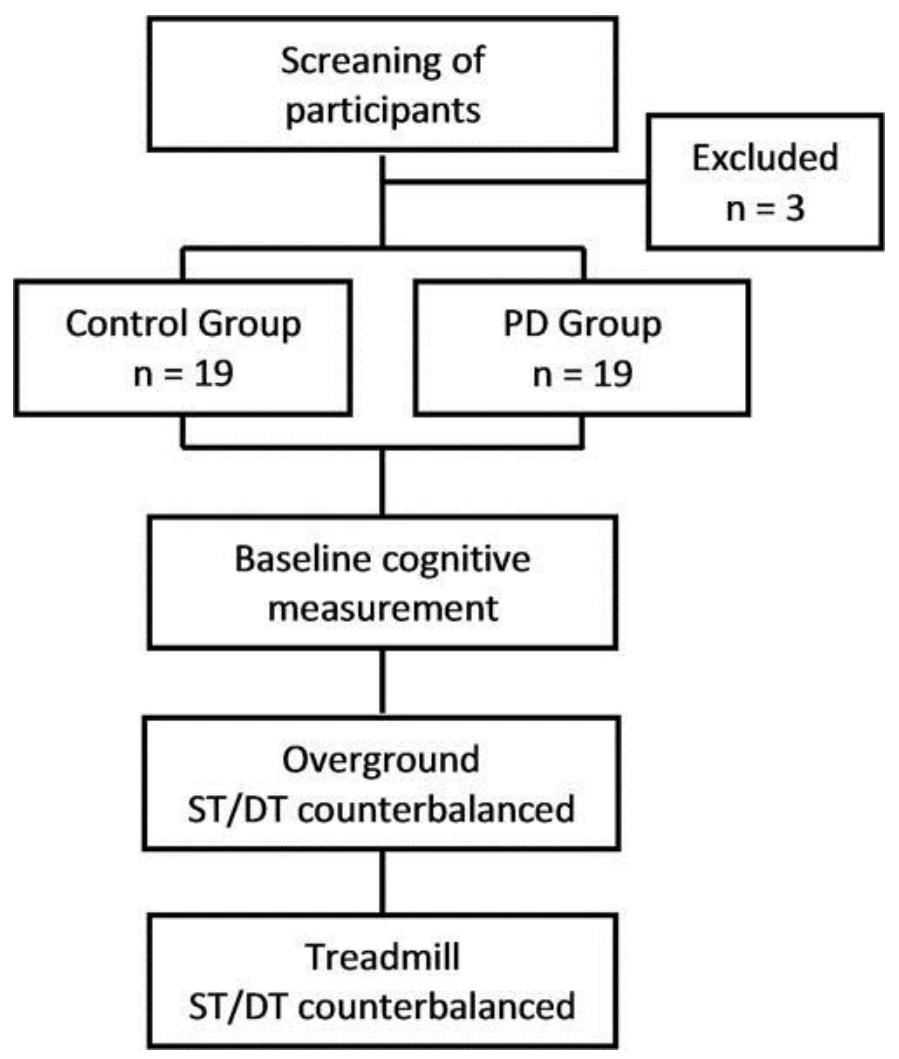

Figure 1. Flowchart of study recruitment and completion

The cognitive task consisted of a phoneme monitoring paradigm. ${ }^{26}$ The phoneme monitoring task has been previously used in several studies of PD and gait. ${ }^{14,27,28}$ Participants were asked to listen to a 1minute-long narration through wireless headphones and they had to count the number of times that two pre-specified words were repeated in the narration. At the end of the task, participants reported the number of times the words were repeated in the text. They were not allowed to use their voice or fingers to help in the count. The subjects were not given any instructions or information regarding prioritization of the tasks. Baseline measurement of the cognitive task (without walking) was performed in a sitting position at the beginning of the session. A total of 14 texts were used, which were arranged in a randomized order for the different cognitive tests (baseline measurement, overground DT, and treadmill DT). The two pre-specified words differed for each text. For the dual task condition, the subjects were not given any instructions or information regarding prioritization of the tasks.

The overground walking tests started with a practical trial of 3 minutes to familiarize the subjects with the walkway and with their self-selected comfortable speed. Participants were tested at their self-selected comfortable speed, up and down an $8-\mathrm{m}$ walkway, for a total time of $1 \mathrm{~min}$. The gait parameters were recorded during the straight walk but not during the turns. Thus, each overground condition (ST or DT) was repeated twice to obtain the same number of steps to that in the treadmill conditions. The only instruction that participants received was to walk at their preferred speed.

The treadmill walking tests started with a period of familiarization of 3 minutes to reach, on the treadmill, the gait speed previously established during the overground condition (ST). During the familiarization period, all participants were instructed to keep their steps close to the front of the treadmill to keep their body erected. The treadmill tests consisted of two new blocks of 3 minutes for ST and DT conditions. During the first minute, belt speed was increased to reach the overground speed. The measurements were taken in the second minute of each block (ST and DT). All participants walked on the treadmill holding the handrails ${ }^{14,20}$ because some subjects, specifically individuals with PD, did not feel safe to walk without the handrail support. Participants walked on the treadmill under the close supervision of a physical therapist. To minimize fatigue effects, rest periods of 3 minutes between treadmill blocks and overground measures were included. 


\section{Data Analysis}

The following variables of gait were evaluated: overground speed $(\mathrm{m} / \mathrm{s})$, stride length $(\mathrm{m})$, stride frequency $(\mathrm{Hz})$, coefficient of variation $(\mathrm{CV})$ of stride length $(\%)$, and $\mathrm{CV}$ of stride frequency $(\%)$. Coefficients of variation were calculated using the following formulas: (standard deviation/stride length) $\times 100$; (standard deviation/stride frequency) $\times 100$. The outcomes of gait measurements were recorded during the tests with OptoGait v.1.9.9.0 software (Microgait, USA) and were exported to Excel format for offline analysis.

The variable of the cognitive task was called "cognitive performance". The cognitive performance was evaluated by calculating the percentage of mistakes made when counting the two pre-specified words.

\section{Statistical Analysis}

To determine group and gait differences, a $\mathrm{t}$ test was performed using the demographic and anthropometric measures. A repeated-measures analysis of variance (ANOVA) was carried out to compare the changes in gait in different conditions. Two-way ANOVA was used for gait speed (analyzed only overground), with "task" as the within-subject factor and "group" as the between-subject factor. For the remaining variables of gait, three-way ANOVA was used with "surface" (overground and treadmill) and "task" (single task and dual task) as within-subject factors and "group" (PD and control) as betweensubject factor. Post hoc t tests were computed. Wilcoxon rank test was applied for each group to compare cognitive performance between baseline, overground, and treadmill conditions. Mann-Whitney test was used to compare cognitive performance between PD patients and controls for baseline, overground, and treadmill conditions. All statistical analysis was performed using PASW Statistics 18. With the exception of percentage of mistakes, none of the data violated the normality assumption according to Shapiro-Wilk test. A P value $<=0.05$ was considered statistically significant.

\section{Results}

$\mathrm{T}$ tests showed no significant differences for age, leg length, weight, and height between PD and control groups (Table 1). Means, standard deviations, and ANOVA results of gait variables are shown in Tables 2 and 3, respectively.

Table 1. Characteristics of PD and control groups

\begin{tabular}{lccc}
\hline & PD & Control & Group Differences \\
\hline Age $(\mathrm{yr})$ & $59.68 \pm 12.81$ & $59.53 \pm 12.10$ & NS \\
Weigh $(\mathrm{kg})$ & $79.91 \pm 14.66$ & $73.63 \pm 8.6$ & NS \\
Leg length $^{\mathrm{a}}(\mathrm{m})$ & $87.37 \pm 7.22$ & $85.64 \pm 6.97$ & NS \\
Height $(\mathrm{m})$ & $1.65 \pm 0.072$ & $1.65 \pm 0.08$ & $\mathrm{NS}$ \\
MMSE & $29.38 \pm 1.02$ & $29.52 \pm 0.89$ & $\mathrm{NS}$ \\
Disease duration $(\mathrm{yr})$ & $5.08 \pm 3.83$ & - & - \\
UPDRS-III & $21.06 \pm 10.69$ & - & - \\
H\&Y stage & $1(\mathrm{n}=4) ; 1.5(\mathrm{n}=3) ; 2(\mathrm{n}=6) ; 2.5(\mathrm{n}=6)$ & - & - \\
(subjects in each stage) & & & \\
\hline
\end{tabular}

Values are mean \pm SD. $t$ test analysis for group differences.

${ }^{a}$ Distance from the great trochanter to the floor.

PD indicates Parkinson disease; MMSE, Mini-Mental State Examination; H\&Y, Hoen and Yahr; UPDRS-III, Unified Parkinson's Disease Rating Scale motor section; NS, not significant; n, sample 
Table 2. Mean \pm standard deviations of gait variables in PD and control groups

\begin{tabular}{|c|c|c|c|c|c|c|c|c|}
\hline & \multicolumn{4}{|c|}{ PD } & \multicolumn{4}{|c|}{ Control } \\
\hline & \multicolumn{2}{|c|}{ Overground } & \multicolumn{2}{|c|}{ Treadmill } & \multicolumn{2}{|c|}{ Overground } & \multicolumn{2}{|c|}{ Treadmill } \\
\hline & ST & DT & ST & DT & ST & DT & ST & DT \\
\hline $\begin{array}{l}\text { Gait speed } \\
(\mathrm{m} / \mathrm{s})\end{array}$ & $1.07 \pm 0.23$ & $1.01 \pm 0.24$ & $1.07 \pm 0.23$ & $1.07 \pm 0.23$ & $1.33 \pm 0.20$ & $1.25 \pm 0.22$ & $1.33 \pm 0.20$ & $1.33 \pm 0.20$ \\
\hline $\begin{array}{l}\text { Stride length } \\
\text { (m) }\end{array}$ & $1.18 \pm 0.20$ & $1.13 \pm 0.20$ & $1.20 \pm 0.23$ & $1.19 \pm 0.24$ & $1.35 \pm 0.17$ & $1.31 \pm 0.17$ & $1.34 \pm 0.18$ & $1.36 \pm 0.16$ \\
\hline $\begin{array}{l}\text { Stride frequency } \\
(\mathrm{Hz})\end{array}$ & $0.90 \pm 0.07$ & $0.93 \pm 0.15$ & $0.90 \pm 0.07$ & $0.93 \pm 0.09$ & $0.98 \pm 0.07$ & $0.95 \pm 0.09$ & $1.00 \pm 0.09$ & $0.98 \pm 0.08$ \\
\hline $\begin{array}{l}\mathrm{CV} \text { of stride length } \\
(\%)\end{array}$ & $4.27 \pm 2.52$ & $4.63 \pm 2.44$ & $1.73 \pm 0.93$ & $1.54 \pm 0.76$ & $2.65 \pm 1.00$ & $3.35 \pm 1.41$ & $1.56 \pm 0.84$ & $1.56 \pm 0.83$ \\
\hline $\begin{array}{l}\text { CV of stride } \\
\text { frequency }(\%)\end{array}$ & $2.60 \pm 0.80$ & $2.96 \pm 0.94$ & $1.90 \pm 1.12$ & $1.88 \pm 1.07$ & $1.84 \pm 0.53$ & $2.37 \pm 0.91$ & $2.91 \pm 1.97$ & $2.80 \pm 2.01$ \\
\hline
\end{tabular}

Values are mean \pm SD.

PD indicates Parkinson disease; ST, single task; DT, dual task; CV, coefficient of variation.

Table 3. ANOVA results of gait variables

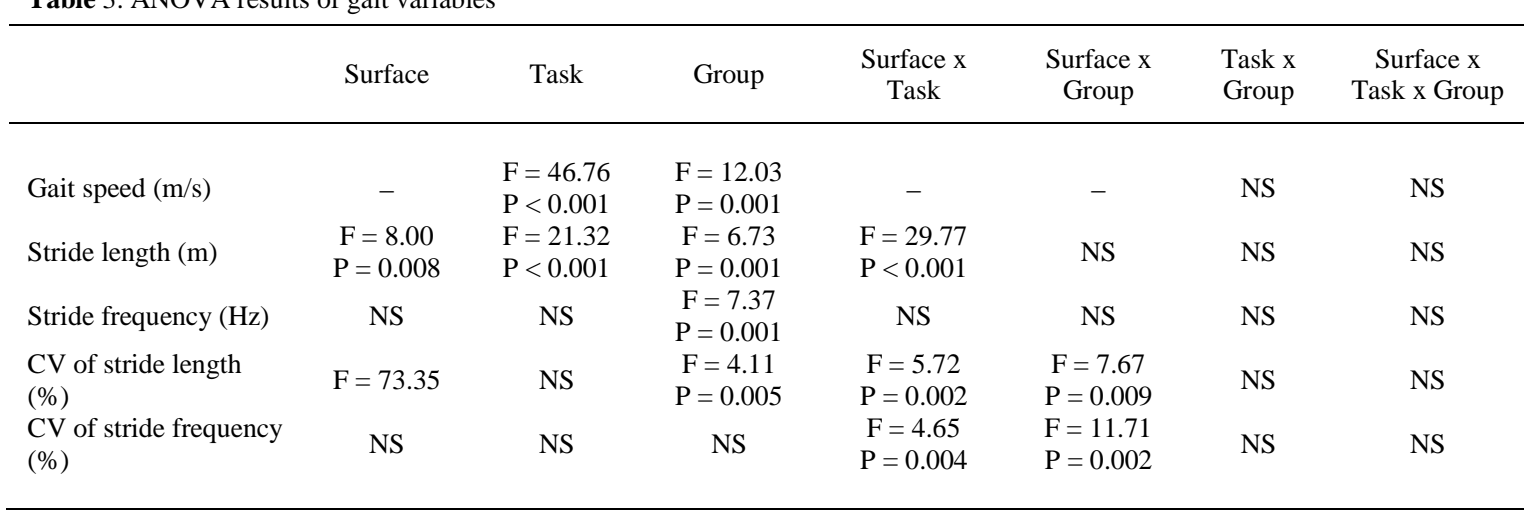

$\mathrm{CV}$ indicates coefficient of variation; NS, not significant

Two-way ANOVA for gait speed showed a significant main effect for group and task factors (Table 3), with higher overground speed in control participants compared with PD patients and also during ST compared with DT. No significant task $\times$ group interaction was found.

Three-way ANOVA for stride frequency showed a significant main effect for group factor (Table 3 ). The control group walked with a higher stride frequency than the PD group.

Three-way ANOVA for stride length revealed a significant surface $\times$ task interaction (Table 3 ). The post hoc analysis showed that for the ST condition, there were no significant differences in stride length between surfaces. However, the stride length was shorter for DT than for ST while walking overground $(\mathrm{F}=44.30 ; \mathrm{P}<0.001)$, but there were no differences between DT and ST conditions during treadmill walking. On the treadmill, stride length for the DT condition was larger than for overground walking $(\mathrm{F}=$ 24.26; P $<0.001)$.

The analysis of the CV of stride frequency showed significant surface $\times$ group and surface $\times$ task interactions (Table 3) (Fig. 2). Therefore, there were no specific group effects of the task in this parameter. The post hoc analysis of the surface $\times$ group interaction showed that overground PD patients walked with a higher $\mathrm{CV}$ of stride frequency than control subjects $(\mathrm{F}=8.67 ; \mathrm{P}=0.006)$. However, $\mathrm{PD}$ patients showed a lower $\mathrm{CV}$ than control subjects while walking on the treadmill $(\mathrm{F}=4.10 ; \mathrm{P}=0.050)$ due to a decrease of the $\mathrm{CV}$ values in the PD group $(\mathrm{F}=6.93 ; \mathrm{P}=0.01)$ and an increase of $\mathrm{CV}$ in control subjects when walking on the treadmill in comparison with walking overground $(\mathrm{F}=4.87 ; \mathrm{P}=0.03)$. The post hoc analysis of the surface $\times$ task interaction indicated that the $\mathrm{CV}$ of stride frequency was higher during DT than during ST in the overground condition $(\mathrm{F}=11.39 ; \mathrm{P}=0.002)$ but not in the treadmill condition. 


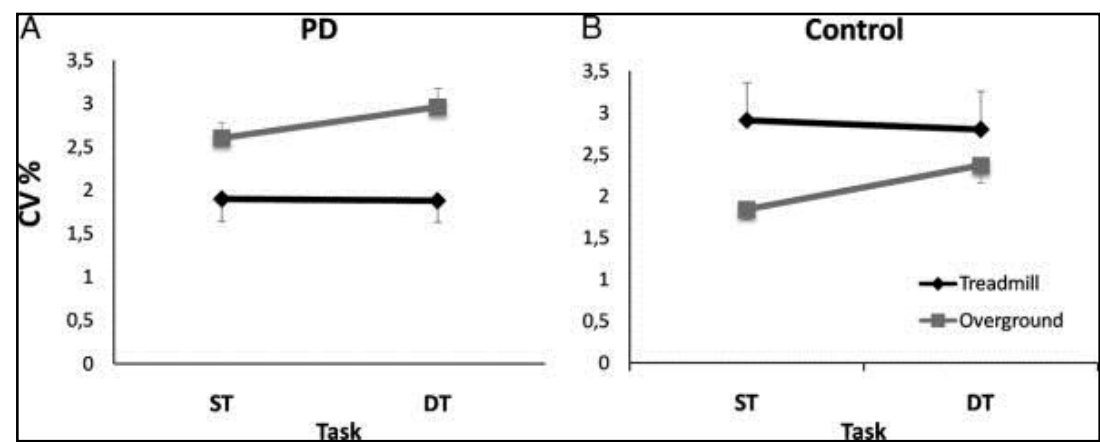

Figure 2 . Comparison of the coefficient of variation of stride frequency between single and dual task, in PD and control groups. PD indicates Parkinson disease; CV, coefficient of variation; ST, single task; DT, dual task.

ANOVA showed significant surface $\times$ group and surface $\times$ task interactions (Table 3 ) for CV of stride length (Fig. 3). These results indicated that there were no specific group effects of the task on the CV of stride length. The post hoc analysis of the surface $\times$ group interaction showed that in overground condition, $\mathrm{PD}$ subjects walked with a higher $\mathrm{CV}$ of stride length compared with control participants $(\mathrm{F}=$ 6.21; $\mathrm{P}=0.017)$. However, these differences were not significant on the treadmill. This was due to a significant reduction of the $\mathrm{CV}$, in both $\mathrm{PD}$ and control subjects, for treadmill versus overground walking $(\mathrm{F}=64.23 ; \mathrm{P}<0.001$ and $\mathrm{F}=16.79 ; \mathrm{P}<0.001)$. The post hoc analysis of the surface $\times$ task interaction showed that the $\mathrm{CV}$ of stride length was higher for DT compared with ST while walking overground $(\mathrm{F}=$ 4.39; $\mathrm{P}=0.04$ ), but there were no significant differences between the tasks on the treadmill. For treadmill walking, the CV was significantly lower than for overground walking for both the ST and DT condition $(\mathrm{F}=48.20 ; \mathrm{P}<0.001$ and $\mathrm{F}=66.45 ; \mathrm{P}<0.001$, respectively).

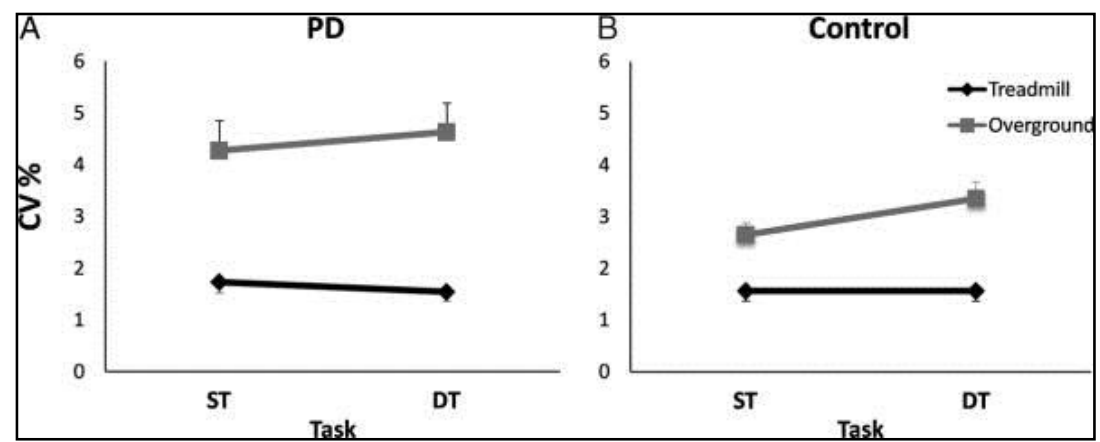

Figure 3 . Comparison of the coefficient of variation of stride length between single and dual task, in PD and control groups. PD indicates Parkinson disease; CV, coefficient of variation; ST, single task; DT, dual task.

The Wilcoxon test in the control group did not show differences on cognitive performance between baseline, overground, and treadmill conditions. However, the PD group showed significant differences between baseline and treadmill $(\mathrm{Z}=2.550 ; \mathrm{P}=0.011)$. PD patients had a greater percentage of mistakes during treadmill walking compared with baseline. No differences were found between baseline and overground $(Z=1.221 ; P=0.222)$ or between overground and treadmill walking $(Z=0.101 ; P=0.92)$ in the PD group. Mann-Whitney tests did not show cognitive differences between groups in the three task conditions. However, there was a tendency for a higher percentage of mistakes in PD participants in comparison with controls during overground $(\mathrm{Z}=1.929 ; \mathrm{P}=0.054)$ and treadmill $(\mathrm{Z}=1.875 ; \mathrm{P}=0.061)$ walking (Fig. 4). 


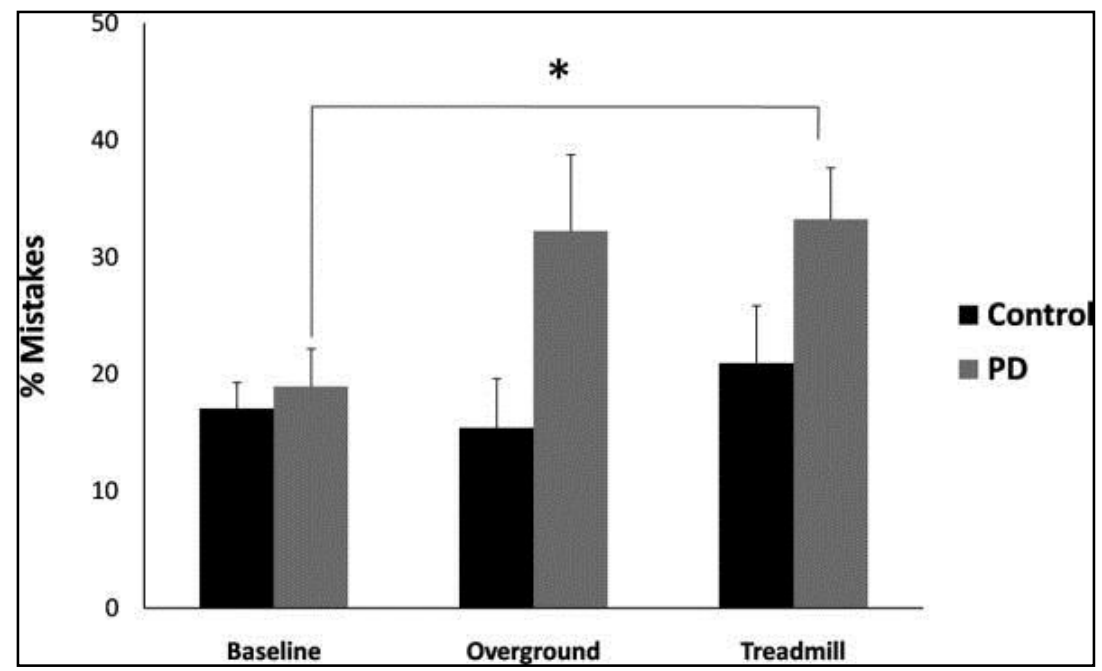

Figure 4 . Comparison of cognitive performance between control and PD subjects for baseline, overground, and treadmill conditions.

\section{Discussion}

The main goal of this study was to explore whether attentional resources may explain the gait improvements observed during treadmill walking in PD. The results show that during treadmill walking with and without the cognitive tasks, individuals with PD reduced their gait variability. These gait improvements did not deteriorate during the performance of a concurrent cognitive task. Moreover, cognitive performance did not show statistical differences between treadmill and overground walking. Therefore, these findings suggest that improvements of gait in PD patients during treadmill walking are independent of attentional demands to the task of walking.

\section{Differences in Gait Parameters Between Surfaces}

The results showed that the PD group demonstrated a shorter stride length, lower velocity, and higher gait variability compared with the control group while walking overground. However, the PD group reduced the $\mathrm{CV}$ of stride frequency and the $\mathrm{CV}$ of stride length when walking on the treadmill. The reduction of the stride frequency variability during treadmill walking has been previously reported in PD. ${ }^{18}$ However, to our knowledge, the current study is the first to report a reduction in the variability of stride length. This is of relevance because a high stride-to-stride variability was associated with an increased risk of falls in PD. ${ }^{29,30}$ Therefore, the present investigation extends the benefits of this device, suggesting that walking on the treadmill could normalize the temporal variability as well as spatial gait variability in PD.

\section{Differences in Gait Between Tasks}

The present data clearly demonstrate that during the performance of a secondary cognitive task, all the participants displayed a decrease in the length of the step and speed together with an increase in the spatial and temporal variability of overground gait. This is in line with previous studies that showed a deterioration of gait disturbances in people with PD when they walk and perform a secondary task at the same time. ${ }^{3,20,22,27}$ Other studies have also shown alterations of the gait pattern in healthy older adults while simultaneously performing a cognitive task, suggesting an age-related reduction in dual task capacity. ${ }^{21,31}$ Moreover, there was a nonsignificant tendency, in PD participants, to perform the cognitive task during walking worse than control subjects. This tendency is in agreement with previous studies showing that PD individuals require more attention to regulate the gait pattern to compensate their damaged automaticity. ${ }^{21}$ Therefore, this report reinforces the hypothesis that walking during the performance of a cognitive task requires greater levels of cognitive function in PD patients. ${ }^{32}$ 
The cognitive task did not affect any gait parameters during treadmill walking in PD and control subjects. Overall, the gait variability reduction in PD participants, as a result of walking on the treadmill, did not deteriorate during the performance of the cognitive task. Therefore, the present study suggests that the adaptative changes of the gait pattern while walking on a treadmill are not related to increased attentional demands in comparison with walking overground.

In the present work, PD patients showed deterioration in their cognitive performance during treadmill walking in comparison with performing a cognitive task in a static position (baseline cognitive measurement). Previous studies showed that when the environment becomes more demanding, focusing on the cognitive task becomes too risky and the attention is allocated toward postural stability to reduce the likelihood of falling and secure safety. ${ }^{33} \mathrm{We}$ cannot rule out the possibility that the handrail support during treadmill walking could "free up" attentional resources related to postural control and stability. Nevertheless, this study suggests that, despite the handrail support, DT dual task on a treadmill could be more demanding for PD participants compared with performing a cognitive task in a rest position without a simultaneous motor task.

\section{Mechanisms of Treadmill Walking in PD}

This article provides additional data to elucidate the role of attentional resource allocation in the treadmill-associated gait improvements observed in patients with PD.

One of the hypotheses to explain the gait improvements in PD subjects is that they are able to allocate more attention to walking on a treadmill than walking overground. ${ }^{19}$ Previous studies showed that people with PD can normalize gait pattern when focusing attention on gait. ${ }^{21}$ However, during the performance of daily activities, distracters impede attention allocation with the consequent gait deterioration.6 During treadmill walking, the environment is more stable and the absence of distracters could help PD subjects to maintain their focus on the gait. The present study does not support this hypothesis. The results reported that $\mathrm{CV}$ of stride frequency was reduced during treadmill walking in both ST and DT conditions (Figs. 23 ). Moreover, an absence of differences in cognitive performance between treadmill and overground walking in PD was observed. Therefore, these findings suggest that attentional demands do not contribute to the gait improvements in PD.

Alternative explanations for the gait improvements are the belt displacement and the constant speed provided by the treadmill. ${ }^{18-20}$ The belt displacement enhances the hip extension movements during treadmill walking. These proprioceptive inputs (repeated contraction and relaxation of muscles groups) may act as an external rhythmical cue bypassing the defective pallidocortical projections, resulting in a normalized gait pattern. ${ }^{20}$ In addition, the propioceptive inputs during treadmill walking could stimulate the Central Pattern Generator (CPG). CPG refers to neural spinal circuits that can produce rhythmic movements. However, the role of $\mathrm{CPG}$ in human control remains unknown. ${ }^{34}$ The constant speed provided by the treadmill could also contribute to minimizing the stride-to-stride variation in gait timing, ${ }^{18}$ in contrast to overground walking where there are ongoing fluctuations in gait speed. The study results showed a reduction in both stride length and frequency variability on the treadmill in PD patients, in comparison with overground. Thus, PD patients could benefit from a constant speed, which improves gait timing. A recent study has suggested that the improvement in the step length may be due to the belt movement but that changes in variability may be influenced further by the imposed treadmill speed. ${ }^{20}$

Although all subjects walked on the treadmill with the handrail support, it is unlikely that this could account for the gait improvement in subjects with PD. A previous study from our group clearly showed that when PD subjects walked with a treadmill simulator without the belt but with hand support, stable environment, and imposed speed, their step length did not improve in comparison with normal overground walking. ${ }^{20}$ Thus, hand support may not be responsible for the gait improvements during treadmill walking that were observed in the present study.

In summary, this is the first study that explores the role of attention in the gait improvements observed in PD subjects during treadmill walking. The present results show that PD subjects walking on a treadmill, while simultaneously performing a cognitive task, are able to maintain the reduction of their gait variability. Moreover, their cognitive execution was similar during treadmill and overground walking. These findings suggest that attentional resources are not involved in the gait treadmill improvements in PD subjects and point to belt displacement and constant speed as alternative explanations. 


\section{Clinical Implications}

The present study provides additional information that may be useful when considering the treadmill as a therapeutic tool to improve the gait in subjects with PD. The reduction of gait variability walking on a treadmill did not deteriorate during the performance of a concurrent cognitive task. Therefore, therapists may include performance of cognitive tasks during the treadmill walking to simulate real-life conditions (ie, walking while keeping a conversation). In addition, we recommend further studies that investigate the role of belt displacement and constant speed in gait improvements using treadmill walking in PD and to determine how these improvements translate to real-life conditions.

\section{Study Limitations}

There are several limitations in this study that must be addressed. First, there were no improvements in the stride length when walking on the treadmill because the severity of the disease of the PD participants in our study was moderate. It has been reported that improvements in stride length walking on a treadmill is specific to advanced PD patients. ${ }^{17}$ Thus, the role of attention over the improvement in the stride length remains unknown. Second, the participants did not show cognitive deficits as assessed by the Mini-Mental State Examination (MMSE). However, this does not exclude possible mild cognitive and attentional deficits in the PD participants. ${ }^{35}$ A comprehensive neuropsychological examination should be used to explore in more detail the executive and cognitive deficits in PD. Third, PD patients were tested only in ON state. ON medication may have impacted cognitive and gait performance because it is known that dual-task walking deficits are improved by anti-Parkinson medications. ${ }^{36}$ Thus, our conclusions may not be applicable to patients in OFF state. Finally, we have only focused on one dual-task model (attention capacity-sharing model). Other types of dual tasking models would help to describe a different cognitive processing that our study is not able to discern. In addition, the use of a more difficult cognitive task could outweigh the "freed-up" attentional demand by the handrail support and help to further delineate differences between conditions and groups.

\section{Conclusions}

The present results suggest that attentional resources do not influence the gait improvements observed in individuals with PD during treadmill walking. This study reinforces the hypothesis that belt displacement and constant speed are the main underlying mechanisms of treadmill walking improvements in PD.

\section{References}

1. Keus SHJ, Munneke M, Nijkrake MJ, et al.: Physical therapy in Parkinson's disease: Evolution and future challenges. Mov Disord 2009; 24: 1-14

2. Dirnberger G, Jahanshahi M: Executive dysfunction in Parkinson's disease: A review. J Neuropsychol 2013; 7: 193-224

3. Yogev-Seligmann G, Hausdorff JM, Giladi N: The role of executive function and attention in gait. Mov Disord 2008; 23: 329-42

4. Yogev G, Giladi N, Peretz C, et al.: Dual tasking, gait rhythmicity, and Parkinson's disease: Which aspects of gait are attention demanding? Eur J Neurosci 2005; 22: 1248-56

5. O'Shea S, Morris ME, Iansek R: Dual task interference during gait in people with Parkinson disease: Effects of motor versus cognitive secondary tasks. Phys Ther 2002; 82: 888-97

6. Rochester L, Hetherington V, Jones D, et al.: Attending to the task: Interference effects of functional tasks on walking in Parkinson's disease and the roles of cognition, depression, fatigue, and balance. Arch Phys Med Rehabil 2004; 85: 1578-85

7. Yogev-Seligmann G, Rotem-Galili Y, Dickstein R, et al.: Effects of explicit prioritization on dual task walking in patients with Parkinson's disease. Gait Posture 2012; 35: 641-6

8. Brauer SG, Morris ME: Can people with Parkinson's disease improve dual tasking when walking? Gait Posture 2010; 31: 229-33

9. Chawla H, Walia S, Behari M, et al.: Effect of type of secondary task on cued gait on people with idiopathic Parkinson's disease. J Neurosci Rural Pract 2014; 5: 18-23

10. Kelly VE, Eusterbrock AJ, Shumway-Cook A: A review of dual-task walking deficits in people with Parkinson's disease: Motor and cognitive contributions, mechanisms, and clinical implications. Parkinsons Dis 2012; 2012: 918719

11. Marois R, Ivanoff J: Capacity limits of information processing in the brain. Trends Cogn Sci 2005; 9: 296-305 
12. Herman T, Giladi N, Gruendlinger L, et al.: Six weeks of intensive treadmill training improves gait and quality of life in patients with Parkinson's disease: A pilot study. Arch Phys Med Rehabil 2007; 88: 1154-8

13. Mehrholz J, Friis R, Kugler J, et al.: Treadmill training for patients with Parkinson's disease. Cochrane Database of Systematic Reviews 2010;Issue 1:CD007830. DOI: 10.1002/14651858.CD007830.pub2

14. Bello O, Sanchez JA, Lopez-Alonso V, et al.: The effects of treadmill or overground walking training program on gait in Parkinson's disease. Gait Posture 2013; 38: 590-5

15. Nadeau A, Pourcher ECP: Effects of 24 wk of treadmill training on gait performance in Parkinson's disease. Med Sci Sport Exerc 2014; 46: 645-55

16. Tseng IJ, Yuan RY, Jeng C: Treadmill training improves forward and backward gait in early Parkinson disease. Am J Phys Med Rehabil 2015;Mar 24 [Epub ahead of print]. doi: 10.1097/PHM.0000000000000273

17. Bello O, Sanchez JA, Fernandez-del-Olmo M: Treadmill walking in Parkinson's disease patients: Adaptation and generalization effect. Mov Disord 2008; 23: 1243-9

18. Frenkel-Toledo S, Giladi N, Peretz C, et al.: Treadmill walking as an external pacemaker to improve gait rhythm and stability in Parkinson's disease. Mov Disord 2005; 20: 1109-14

19. Bello O, Fernandez-Del-Olmo M: How does the treadmill affect gait in Parkinson's disease? Curr Aging Sc 2012; 5: 28-34

20. Bello O, Marquez G, Camblor M, et al.: Mechanisms involved in treadmill walking improvements in Parkinson's disease. Gait Posture 2010; 32: 118-23

21. Morris ME, Iansek R, Matyas TA, et al.: Stride length regulation in Parkinson's disease. Normalization strategies and underlying mechanisms. Brain 1996; 119 (pt 2): 551-68

22. Canning CG: The effect of directing attention during walking under dual-task conditions in Parkinson's disease. Parkinsonism Relat Disord 2005; 11: 95-9

23. Hughes AJ, Daniel SE, Kilford L, et al.: Accuracy of clinical diagnosis of idiopathic Parkinson's disease: A clinico-pathological study of 100 cases. J Neurol Neurosurg Psychiatry 1992; 55: 181-4

24. Fahn S, Elton R, Fahn S, et al., editors. Members of the UPDRS development committee. Recent developments in Parkinson's disease, vol. 2. Florham Park, NJ: MacMillan Health Care Information; 1987

25. Hoehn MM, Yahr MD: Parkinsonism: Onset, progression and mortality. Neurology 1967; 17: 427-42

26. Connine CM: Phoneme monitoring. Lang Cogn Process 1996; 11: 635-45

27. Springer S, Giladi N, Peretz C, et al.: Dual-tasking effects on gait variability: The role of aging, falls, and executive function. Mov Disord 2006; 21: 950-7

28. Wild L, de Lima D, Balardin J, et al.: Characterization of cognitive and motor performance during dual-tasking in healthy older adults and patients with Parkinson's disease. J Neurol 2013; 260: 580-9

29. Hausdorff JM, Lowenthal J, Herman T, et al.: Rhythmic auditory stimulation modulates gait variability in Parkinson's disease. Eur J Neurosci 2007; 26: 2369-75

30. Schaafsma JD, Giladi N, Balash Y, et al.: Gait dynamics in Parkinson's disease: Relationship to Parkinsonian features, falls and response to levodopa. J Neurol Sci 2003; 212: 47-53

31. Rochester L, Galna B, Lord S, et al.: The nature of dual-task interference during gait in incident Parkinson's disease. Neuroscience 2014; 265: 83-94

32. Plotnik M, Giladi N, Dagan Y, et al.: Postural instability and fall risk in Parkinson's disease: Impaired dual tasking, pacing, and bilateral coordination of gait during the "oN" medication state. Exp Brain Res 2011; 210: $529-38$

33. Yogev-Seligmann G, Hausdorff JM, Giladi N: Do we always prioritize balance when walking? Towards an integrated model of task prioritization. Mov Disord 2012; 27: 765-70

34. Dietz V: Spinal cord pattern generators for locomotion. Clin Neurophysiol 2003; 114: 1379-89

35. Chou KL, Lenhart A, Koeppe RA, et al.: Abnormal MoCA and normal range MMSE scores in Parkinson disease without dementia: Cognitive and neurochemical correlates. Parkinsonism Relat Disord 2014; 20: 1076-80

36. Lord S, Baker K, Nieuwboer A, et al.: Gait variability in Parkinson's disease: An indicator of non-dopaminergic contributors to gait dysfunction? J Neurol 2011; 258: 566-72 